\title{
Normal periodic solutions for the fractional abstract Cauchy problem
}

\author{
Jennifer Bravo ${ }^{1}$ and Carlos Lizama ${ }^{1 *}$ (1)
}

\section{"Correspondence:}

carlos.lizama@usach.cl

'Departamento de Matemática y

Ciencia de la Computación,

Universidad de Santiago de Chile,

Las Sophoras 173, Estación Central,

Santiago, Chile

\section{Springer}

\begin{abstract}
We show that if $A$ is a closed linear operator defined in a Banach space $X$ and there exist $t_{0} \geq 0$ and $M>0$ such that $\left\{(i m)^{\alpha}\right\}_{|m|>t_{0}} \subset \rho(A)$, the resolvent set of $A$, and

$$
\left\|(i m)^{\alpha}\left(A+(i m)^{\alpha} l\right)^{-1}\right\| \leq M \quad \text { for all }|m|>t_{0}, m \in \mathbb{Z},
$$

then, for each $\frac{1}{p}<\alpha \leq \frac{2}{p}$ and $1<p<2$, the abstract Cauchy problem with periodic boundary conditions

$$
\left\{\begin{array}{l}
{ }_{G L} D_{t}^{\alpha} u(t)+A u(t)=f(t), \quad t \in(0,2 \pi) ; \\
u(0)=u(2 \pi),
\end{array}\right.
$$

where ${ }_{G L} D^{\alpha}$ denotes the Grünwald-Letnikov derivative, admits a normal $2 \pi$-periodic solution for each $f \in L_{2 \pi}^{p}(\mathbb{R}, X)$ that satisfies appropriate conditions. In particular, this happens if $A$ is a sectorial operator with spectral angle $\phi_{A} \in(0, \alpha \pi / 2)$ and $\int_{0}^{2 \pi} f(t) d t \in \operatorname{Ran}(A)$.

MSC: 35R11; 35B10; 43A50

Keywords: Grünwald-Letnikov fractional order derivative; Periodic solution; Normal convergence
\end{abstract}

\section{Introduction}

Existence of periodic solutions for differential equations of fractional order is a very desirable property for analyzing cyclic (e.g. biological) processes, see [28]. In recent years many papers have appeared on this topic $[1,2,17,23]$, and there are different methods that allow periodic solutions, the Fourier transform being the most common. On the other hand, it is well known that we cannot expect the existence of periodic solutions in time-invariant systems with each definition of fractional order derivative, see e.g. [24, 26, 27].

Regarding the fractional abstract Cauchy problem

$$
\left\{\begin{array}{l}
{ }_{G L} D_{t}^{\alpha} u(t)+A u(t)=f(t), \quad t \in(0,2 \pi) ; \\
u(0)=u(2 \pi),
\end{array}\right.
$$

(c) The Author(s) 2021. This article is licensed under a Creative Commons Attribution 4.0 International License, which permits use, sharing, adaptation, distribution and reproduction in any medium or format, as long as you give appropriate credit to the original author(s) and the source, provide a link to the Creative Commons licence, and indicate if changes were made. The images or other third party material in this article are included in the article's Creative Commons licence, unless indicated otherwise in a credit line to the material. If material is not included in the article's Creative Commons licence and your intended use is not permitted by statutory regulation or exceeds the permitted use, you will need to obtain permission directly from the copyright holder. To view a copy of this licence, visit http://creativecommons.org/licenses/by/4.0/. 
where $A$ is a closed linear operator defined in a Banach space $X$ and ${ }_{G L} D^{\alpha}$ denotes the Grünwald-Letnikov derivative, the authors in $[3,22]$ used a method based on operatorvalued Fourier multipliers to obtain existence of periodic solutions, in several senses. With this method they obtained solutions belonging to the periodic Lebesgue space $L_{2 \pi}^{p}(\mathbb{R}, X)$, where $X$ is a Banach space. More precisely, assuming that $1<\alpha \leq 2$ and $X$ satisfy a geometrical hypothesis, in [22, Theorem 3.15] the authors showed that for all $f \in L_{2 \pi}^{p}(\mathbb{R}, X)$ there exists unique $u \in H_{2 \pi}^{\alpha, p}(\mathbb{R}, X) \cap L_{2 \pi}^{p}(\mathbb{R}, D(A))$ satisfying (1) if and only if $\left\{(i m)^{\alpha}\right\}_{m \in \mathbb{Z}} \subseteq \rho(A)$ and the set $\left\{(i m)^{\alpha}\left(A+(i m)^{\alpha} I\right)^{-1}\right\}_{m \in \mathbb{Z}}$ is Rademacher bounded (or $R$-bounded). See also [3, Theorem 3.3] for the analogous result in the case $0<\alpha \leq 1$ and $[5-7,18]$ for extensions of this result to more general models.

However, this method has disadvantages in concrete applications because they require checking the $R$-boundedness condition on the operator-valued symbol. An additional problem of the characterization cited above is the implicit requirement that $0 \in \rho(A)$, which restricts the applicability of the result. For instance, the case where $A$ is the Laplacian operator defined on unbounded domains cannot be considered by the above characterization.

To avoid these difficulties, some authors [4] proposed the sum method [8] that was first introduced by Da Prato and Grisvard in the context of sectorial operators. The main idea is to transform the problem into the closedness of the sum of two closed operators related to (1). However, although the $R$-boundedness condition is unnecessary with this method, it only allows to establish the existence of periodic solutions in some proper subspaces of $L_{2 \pi}^{p}(\mathbb{R}, X)$, see [4, Theorem 1$]$.

In this article we take a different approach. Starting from the observation that periodic solutions of systems are usually represented by a series formed by a set of functions, the present work introduces a novel concept of solution that implies the formal representation of the solution by means of normally convergent series. This new concept is general enough to admit periodic forcing terms in the space $L_{2 \pi}^{p}(\mathbb{R}, X)$ without assuming any geometrical conditions in $X$ or $R$-boundedness of the operator-valued symbol. It allows also to avoid assuming a fortiori that $0 \in \rho(A)$. We note that Haraux [16, Chapter B, I] gave a similar approach in the case that $X$ is a Hilbert space, which has been the main motivation in this work.

Using this approach, we can capture the minimum requirements that are needed in a system of $2 \pi$-periodic solutions of (1) in the sense that any solution of (1) can be represented by a normally convergent series formed by functions of the following set:

$$
\left\{u_{m} e^{i m t}\right\}_{|m| \leq t_{0}} \cup\left\{\left(A+(i m)^{\alpha} I\right)^{-1} \widehat{f}_{m} e^{i m t}\right\}_{|m|>t_{0}},
$$

where we assume $\left\{(i m)^{\alpha}\right\}_{|m|>t_{0}} \subseteq \rho(A)$ and $\hat{f}_{m}=\left(\left(A+(i m)^{\alpha} I\right)^{-1}\right) u_{m}$ for $|m| \leq t_{0}$, where $\widehat{f}_{m}$ are the Fourier coefficients of $f$. It is notable that the exact value of $t_{0}$ can be determined explicitly in some important examples. For example, assuming that $A=B+C$, where $C$ is a bounded operator, we consider two situations of interest. First, when $X$ is a Hilbert space and $B$ is selfadjoint, then we have $t_{0}=\frac{2^{1 / \alpha}\|C\|^{1 / \alpha}}{|\sin (\alpha \pi / 2)|^{1 / \alpha}}$. Second, when $X$ is a Banach space and $B$ is sectorial with spectral angle $0<\phi<\alpha \pi / 2$, then we have $t_{0}=2^{1 / \alpha}\|C\|^{1 / \alpha}$. We will show the validity of these criteria for all pairs $(p, \alpha)$ belonging to the sector

$$
\left\{p \in(1,2]: \frac{1}{p}<\alpha \leq \frac{2}{p}\right\}
$$


This article is organized as follows: Sect. 2 is devoted to recalling some preliminaries, including the definition and the main properties of the Grünwald-Letnikov derivative. The notions of sectoriality of a closed linear operator and normally convergent series close this short section. Section 3 introduces the notion of normal periodic solution and contains our main result (Theorem 3.2). After that, two important consequences are shown. The first takes the additive perturbation of the operator $A$ in (1) as the sum of a selfadjoint operator defined in a Hilbert space and a bounded linear operator. The second takes the additive perturbation again but now in the scenario of a Banach space. In this case, we assume that $A$ can be represented as a sum of a sectorial operator with an angle depending on the fractional parameter $\alpha$ and a bounded linear operator. In both cases, we can guarantee the existence of normal $2 \pi$-periodic solutions.

\section{Preliminaries}

In this section we recall some preliminary results and definitions that will be used throughout the paper.

Let $X$ be a complex Banach space. Given $1 \leq p<\infty$, we consider the Banach space $L_{2 \pi}^{p}(\mathbb{R}, X)$ of $X$-valued, $2 \pi$-periodic measurable functions $f$ on $\mathbb{R}$ such that

$$
\|f\|_{p}:=\left(\frac{1}{2 \pi} \int_{0}^{2 \pi}\|f(t)\|^{p} d t\right)^{1 / p}<\infty
$$

For a function $f \in L_{2 \pi}^{1}(\mathbb{R}, X)$, we denote by $\hat{f}_{k}, k \in \mathbb{Z}$, the $k$ th Fourier coefficient of $f$ :

$$
\hat{f}_{k}=\frac{1}{2 \pi} \int_{0}^{2 \pi} e^{-i k t} f(t) d t, \quad k \in \mathbb{Z} .
$$

Let $X, Y$ be Banach spaces. We denote by $\mathcal{B}(X, Y)$ the space of all bounded linear operators from $X$ to $Y$. When $X=Y$, we write simply $\mathcal{B}(X)$. For a linear operator $A$ on $X$, we denote the domain by $D(A)$ and its resolvent set by $\rho(A)$, and for $\lambda \in \rho(A)$, we write $R(\lambda, A)=$ $(\lambda I-A)^{-1}$. By $[D(A)]$ we denote the domain of $A$ equipped with the graph norm.

We recall the well-known definition of the Grünwald-Letnikov fractional derivative and some of its properties presented in [12, 20, 21, 25], see [25, Sect. 2.3, p. 6]. Let $\alpha>0$. Given $f \in L_{2 \pi}^{p}(\mathbb{R}, X), 1 \leq p<\infty$, the Riemann difference

$$
\left(\Delta_{h}^{\alpha} u\right)(t)=\sum_{j=0}^{\infty} k^{-\alpha}(j) u(t-j h),
$$

where $k^{-\alpha}(n)=\frac{\Gamma(-\alpha+n)}{\Gamma(-\alpha) \Gamma(-\alpha+n)}, n \in \mathbb{N}_{0}$, satisfy

$$
\sum_{j=0}^{\infty} k^{-\alpha}(j) z^{j}=(1-z)^{\alpha}, \quad|z|<1,
$$

exists almost everywhere and

$$
\left\|\Delta_{h}^{\alpha} u\right\|_{p} \leq\|u\|_{p} \sum_{j=0}^{\infty}\left|k^{-\alpha}(j)\right|<\infty
$$


since $k^{-\alpha}(n)=\frac{1}{n^{1+\alpha} \Gamma(\alpha)}\left(1+O\left(\frac{1}{n}\right)\right)$. For a detailed study of the sequence $k^{\beta}$ and its properties, we refer the reader to the recent article [13].

The following definition was proposed in [22, Definition 2.1].

Definition 2.1 Let $X$ be a complex Banach space, $\alpha>0$, and $1 \leq p<\infty$. If for $f \in L_{2 \pi}^{p}(\mathbb{R}, X)$ there exists $g \in L_{2 \pi}^{p}(\mathbb{R}, X)$ such that $\lim _{h \rightarrow 0^{+}} \frac{\Delta_{h}^{\alpha} f}{h^{\alpha}}=g$ in the $L^{p}$-norm, then $g$ is called the $\alpha$ th Grünwald-Letnikov derivative of $f$. We use the notation $g={ }_{G L} D^{\alpha} f$.

Remark 2.2 It should be noted that in [26] it was shown that the fractional-order derivative, based on the Grünwald-Letnikov definition, of a periodic function with a specific period cannot be a periodic function with the same period. However, the definition of the Grünwald-Letnikov fractional derivative considered in [26, Formula (1)] differs from ours, which is taken from the book by Samko, Kilbas, and Marichev [20, Sect. 20, p. 371] and which, in turn, coincides with the Marchaud derivative [20, Theorem 20.2]. See also [19] for an approach in the context of periodic distributions.

The next result shows some examples and properties. For a proof, see e.g. [12, Propositions 9, 11, and 12] and [22, Proposition 2.3].

Proposition 2.3 Let $f \in L_{2 \pi}^{p}(\mathbb{R}, X), 1 \leq p<\infty$. For any $z \in \mathbb{C}, \operatorname{Re}(z) \geq 0, \alpha, \beta>0$, and $x, t \in \mathbb{R}$, we have

(i) ${ }_{G L} D_{t}^{\alpha} e^{z t}=z^{\alpha} e^{z t}$;

(ii) ${ }_{G L} D_{t}^{\alpha} \sin (x t)=x^{\alpha} \sin \left(x t+\alpha \frac{\pi}{2}\right)$ and ${ }_{G L} D_{t}^{\alpha} \cos (x t)=x^{\alpha} \cos \left(x t+\alpha \frac{\pi}{2}\right)$;

(iii) If ${ }_{G L} D^{\alpha} f \in L_{2 \pi}^{p}(\mathbb{R}, X)$, then ${ }_{G L} D^{\beta} f \in L_{2 \pi}^{p}(\mathbb{R}, X)$ for all $0<\beta<\alpha$;

(iv) ${ }_{G L} D_{G L}^{\alpha} D^{\beta} f={ }_{G L} D^{\alpha+\beta} f$ whenever one of the two sides is well defined.

We recall the definition of normal convergence for a series of functions [14, Definition 3 , p. 222 ], [10, 6.19, p. 64] and its relationship with uniform convergence [14, Theorem 1, p. 222], [10, Theorem 6.1.10, p. 64].

Definition 2.4 Given a set $I$ and bounded functions $u_{n}: I \rightarrow X$, the series $\sum_{n \in \mathbb{Z}} u_{n}(t)$ is called normally convergent on $I$ if the series $\sum_{n \in \mathbb{Z}}\left\|u_{n}\right\|_{\infty}:=\sum_{n \in \mathbb{Z}} \sup _{t \in I}\left\|u_{n}(t)\right\|$ converges.

Remark 2.5 Every normal convergent series is uniformly convergent.

Let $\Sigma_{\phi} \subset \mathbb{C}$ be the open sector $\Sigma_{\phi}=\{\lambda \in \mathbb{C} \backslash\{0\}:|\arg \lambda|<\phi\}$. Finally, we recall the following definition.

Definition 2.6 ([9]) Given a closed linear operator $A$ in $X$, we say that $A$ is sectorial if $A$ satisfies the following conditions (i) $\overline{D(A)}=X, \overline{R(A)}=X,(-\infty, 0) \subset \rho(A)$; (ii) $\left\|t(t+A)^{-1}\right\| \leq$ $M$ for all $t>0$ and some $M>0$. The operator $A$ is called $R$-sectorial if the set $\left\{t(t+A)^{-1}\right\}_{t>0}$ is $R$-bounded.

If $A$ is sectorial, then $\Sigma_{\phi} \subset \rho(-A)$ for some $\phi>0$ and $\sup _{|\arg \lambda|<\phi}\left\|\lambda(\lambda+A)^{-1}\right\|<\infty$. We denote the spectral angle of a sectorial operator $A$ by

$$
\phi_{A}=\inf \left\{\phi: \Sigma_{\pi-\phi} \subset \rho(-A), \sup _{\lambda \in \Sigma_{\pi-\phi}}\left\|\lambda(\lambda+A)^{-1}\right\|<\infty\right\} .
$$




\section{Main results}

Let $X$ be a complex Banach space and $A: D(A) \subset X \rightarrow X$ be a closed linear operator on $X$. Given $\alpha>0$ and $f \in L_{2 \pi}^{p}(\mathbb{R}, X)$, we are concerned with the problem of existence of periodic solutions of the equation

$$
\left\{\begin{array}{l}
{ }_{G L} D_{t}^{\alpha} u(t)+A u(t)=f(t), \quad t \in(0,2 \pi), \\
u(0)=u(2 \pi) .
\end{array}\right.
$$

Let $x \in X$ be fixed. Define $f_{m}(t):=e^{i m t} x, m \in \mathbb{Z}$. It is clear that $f_{m} \in L_{2 \pi}^{p}(\mathbb{R}, X)$ for any $p>1$. Suppose that $x \in \operatorname{Ran}\left(A+(i n)^{\alpha} I\right)$ for some $\alpha>0$ and some $n \in \mathbb{Z}$ fixed. Here

$$
(\text { in })^{\alpha}:=|n|^{\alpha} e^{\frac{1}{2} \operatorname{sgn}(n) i \pi \alpha}
$$

Then defining $u_{n}(t):=e^{i n t} u_{n}$ where $u_{n} \in D(A)$ is such that $x=\left(A+(i n)^{\alpha} I\right) u_{n}$, and in view of Proposition 2.3 (part (i)), we obtain

$$
{ }_{G L} D_{t}^{\alpha} u_{n}(t)+A u_{n}(t)=(i n)^{\alpha} e^{i n t} u_{n}+e^{i n t} A u_{n}=e^{i n t}\left((i n)^{\alpha} I+A\right) u_{n}=f_{n}(t) .
$$

In other words, $u_{n}(t)$ is a strict (or strong) $2 \pi$-periodic solution of (5).

Motivated by the previous example and the concept of a normal convergent series, we present the following definition.

Definition 3.1 We say that a sequence of functions $u_{m}: \mathbb{R} \rightarrow X, m \in \Lambda \subseteq \mathbb{Z}$ is a normal $2 \pi$-periodic solution of (5) if $u_{m}$ is $2 \pi$-periodic, $u_{m}(t) \in D(A)$ for all $t \in \mathbb{R}$ and satisfies (5) for each $m \in \Lambda$ and the series $\sum_{n \in \mathbb{Z}} \mathbf{1}_{\Lambda}(n) u_{n}(t)$ is normally convergent.

In the above definition, we have $\mathbf{1}_{\Lambda}(n) \equiv 1$ if $n \in \Lambda$ and 0 in the other case. Our main result is the following theorem.

Theorem 3.2 Suppose that there exist $t_{0} \geq 0$ and $M>0$ such that $\left\{(\text { im })^{\alpha}\right\}_{|m|>t_{0}} \subset \rho(A)$ and

$$
\|(\text { im })^{\alpha}\left(A+(\text { im })^{\alpha} I\right)^{-1} \| \leq M \quad \text { for all }|m|>t_{0}, m \in \mathbb{Z} .
$$

If $\in L_{2 \pi}^{p}(\mathbb{R}, X)$, where $1<p \leq 2$, and there exists $u_{m} \in D(A)$ such that $\hat{f}_{m}=\left(A+(\text { im })^{\alpha} I\right) u_{m}$ for each $|m| \leq t_{0}$, then the sequence of partial sums

$$
u_{N}(t):=\sum_{|m| \leq t_{0}} u_{m} e^{i m t}+\sum_{t_{0}<|m| \leq N}\left(A+(i m)^{\alpha} I\right)^{-1} \widehat{f}_{m} e^{i m t}, \quad N>t_{0},
$$

is a normal $2 \pi$-periodic solution of (5) for all $\frac{1}{p}<\alpha \leq \frac{2}{p}$.

Proof Let $N>t_{0}$ be fixed, and define

$$
f_{N}(t):=\sum_{m=-N}^{N} \widehat{f}_{m} e^{i m t}
$$


and

$$
v_{t_{0}}(t):=\sum_{|m| \leq t_{0}} u_{m} e^{i m t}
$$

Note that by Proposition 2.3 we have

$$
\begin{aligned}
{ }_{G L} D_{t}^{\alpha}\left(e^{i m t} u_{m}\right)+A\left(e^{i m t} u_{m}\right) & =(i m)^{\alpha} e^{i m t} u_{m}+e^{i m t} A u_{m} \\
& =e^{i m t}\left((i m)^{\alpha} I+A\right) u_{m}=e^{i m t} \widehat{f}_{m}
\end{aligned}
$$

We conclude that $v_{t_{0}}$ is a $2 \pi$-periodic solution of (5). On the other hand, the identity

$$
A\left(A+(i m)^{\alpha} I\right)^{-1}+(i m)^{\alpha}\left(A+(i m)^{\alpha} I\right)^{-1}=I
$$

shows that

$$
\begin{aligned}
{ }_{G L} & D_{t}^{\alpha}\left[\left(A+(i m)^{\alpha} I\right)^{-1} \widehat{f}_{m} e^{i m t}\right]+A\left[\left(A+(i m)^{\alpha} I\right)^{-1} \widehat{f}_{m} e^{i m t}\right] \\
& =(i m)^{\alpha}\left(A+(i m)^{\alpha} I\right)^{-1} \widehat{f}_{m} e^{i m t}+A\left(A+(i m)^{\alpha} I\right)^{-1} \widehat{f}_{m} e^{i m t} \\
& =\left[(i m)^{\alpha}\left(A+(i m)^{\alpha} I\right)^{-1}+A\left(A+(i m)^{\alpha} I\right)^{-1}\right] \widehat{f}_{m} e^{i m t} \\
& =\widehat{f}_{m} e^{i m t}
\end{aligned}
$$

Therefore, we can conclude that ${ }_{G L} D_{t}^{\alpha} u_{N}(t)+A u_{N}(t)=f_{N}(t)$ i.e. $u_{N}$ is a $2 \pi$-periodic solution of (5).

Now, we study the convergence normal of the series. As a consequence of (6), we have, for $|m|>t_{0}$,

$$
\left\|\left(A+(i m)^{\alpha} I\right)^{-1} \widehat{f}_{m} e^{i m t}\right\|_{\infty}=\sup _{t \in \mathbb{T}}\left\{\left\|\left(A+(i m)^{\alpha} I\right)^{-1} \widehat{f}_{m} e^{i m t}\right\| \leq \frac{M\left\|\widehat{f}_{m}\right\|}{|m|^{\alpha}} .\right.
$$

On the other hand, by a theorem of Hardy and Littlewood (see e.g. [11]) we know that there exists a constant $C>0$ such that

$$
\left(\sum_{m \in \mathbb{Z}}|m|^{p-2}\left\|\widehat{f}_{m}\right\|^{p}\right)^{1 / p} \leq C\|f\|_{p}
$$

for each $1<p \leq 2$. Hence, by Hölder's inequality, we obtain for any $1 \leq q<\infty$ with $\frac{1}{p}+\frac{1}{q}=1$

$$
\begin{aligned}
\sum_{|m|>t_{0}} \frac{M\left\|\widehat{f}_{m}\right\|}{|m|^{\alpha}} & \leq M \sum_{m \in \mathbb{Z} \backslash\{0\}} \frac{|m|^{(p-2) / p} \mid \widehat{f}_{m} \|}{|m|^{\alpha+(p-2) / p}} \\
& \leq M C\left(\sum_{m \in \mathbb{Z} \backslash\{0\}}|m|^{p-2} \mid \widehat{f}_{m} \|^{p}\right)^{1 / p}\left(\sum_{m \in \mathbb{Z} \backslash\{0\}} \frac{1}{|m|^{q(\alpha+(p-2) / p)}}\right)^{1 / q} .
\end{aligned}
$$

Since $1<\alpha p \leq 2$, we obtain $q(\alpha+(p-2) / p)>1$, and the result follows.

For instance, in case $p=2$ the restriction is: $\frac{1}{2}<\alpha \leq 1$. A complete picture is given in Fig. 1. 


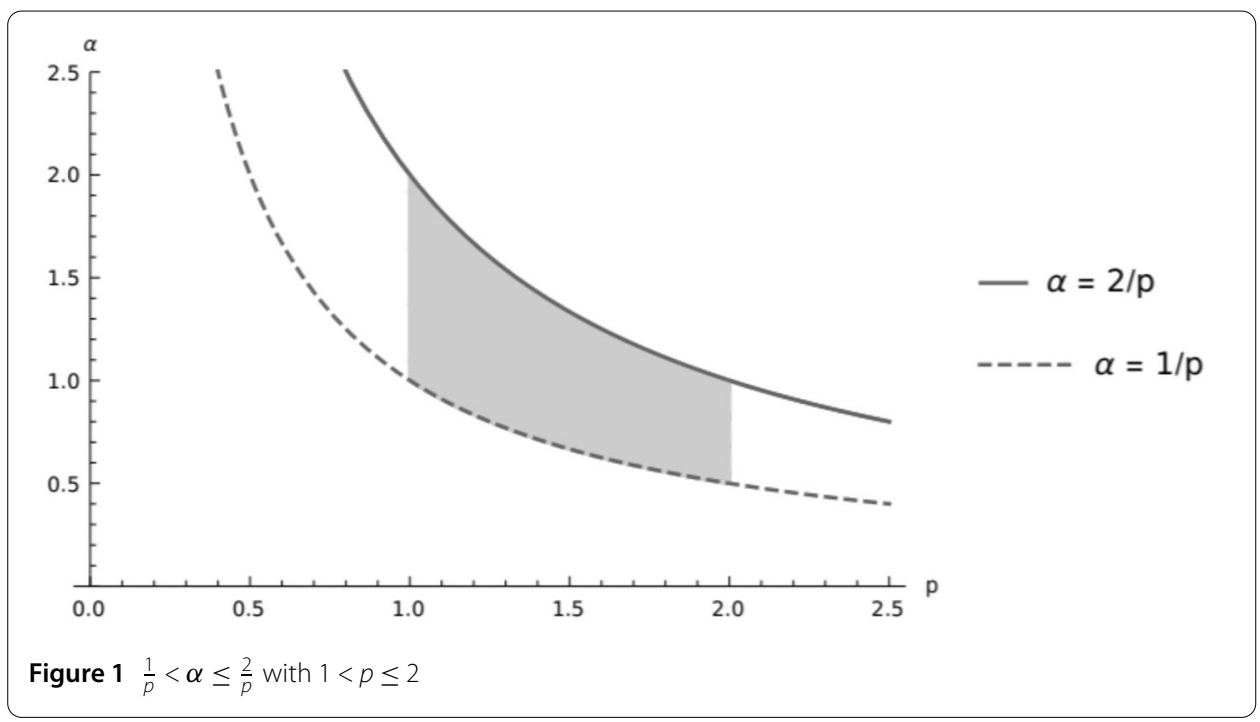

An immediate consequence of Theorem 3.2 is the following.

Corollary 3.3 Let $A \in \mathcal{B}(X)$ and $1<p \leq 2$. If $f \in L_{2 \pi}^{p}(\mathbb{R}, X)$ satisfies that $\widehat{f}_{m} \in \operatorname{Ran}(A+$ $\left.(\text { im })^{\alpha} I\right)$ for $|m| \leq\|A\|^{1 / \alpha}$, where $\frac{1}{p}<\alpha \leq \frac{2}{p}$, then the conclusions of Theorem 3.2 hold.

Proof Since $A$ is bounded, we have that $\lambda \in \rho(A)$ for all $|\lambda|>\|A\|$ and

$$
(\lambda+A)^{-1}=\sum_{k=0}^{\infty} \frac{A^{k}}{\lambda^{k+1}}
$$

Choosing $t_{0}:=\|A\|^{\frac{1}{\alpha}}$ and $\lambda=(i t)^{\alpha}$, we obtain for each $|t|>t_{0}$ that $\left\{(i t)^{\alpha}\right\}_{|t|>t_{0}} \subset \rho(A)$ and $\left\|\left((i t)^{\alpha}+A\right)^{-1}\right\|=\left\|(\lambda+A)^{-1}\right\| \leq \frac{e}{|\lambda|}=\frac{M}{|t|^{\alpha}}$ for each $|t|>t_{0}$, where $M:=e$.

In the case of unbounded operators on Hilbert spaces, we have the following result that generalizes and improves [16, Chapter B, Lecture 20, Corollary 10, p. 157].

Theorem 3.4 Let $B$ a selfadjoint operator with domain $D(B)$ defined on a Hilbert space $H$ and $C \in \mathcal{B}(H)$. Assume that $B$ commutes with $C$, and let $A=B+C$. Let $1<p \leq 2$. Iff $\in$ $L_{2 \pi}^{p}(\mathbb{R}, H)$ satisfies that $\widehat{f}_{m} \in \operatorname{Ran}\left(A+(\text { im })^{\alpha} I\right)$ for $|m| \leq \frac{2^{1 / \alpha}\|C\|^{1 / \alpha}}{|\sin (\alpha \pi / 2)|^{1 / \alpha}}, m \in \mathbb{Z}$, where $\frac{1}{p}<\alpha \leq \frac{2}{p}$, then the conclusions of Theorem 3.2 hold.

Proof Let $s \in \mathbb{R}$ such that $|s|>s_{0}:=\frac{2^{1 / \alpha}\|C\|^{1 / \alpha}}{|\sin (\alpha \pi / 2)|^{1 / \alpha}}$. Since $B$ is selfadjoint, we have $\sigma(B) \subset \mathbb{R}$ and

$$
\left\|(B+\lambda I)^{-1}\right\| \leq \frac{1}{|\operatorname{Im}(\lambda)|}
$$

for all $\lambda \in \mathbb{C} \backslash \mathbb{R}$ (see e.g. [15, Proposition C.4.2, p. 321]). In particular, choosing $\lambda=(i s)^{\alpha}$, we obtain that $\left\{(i s)^{\alpha}\right\}_{|s|>s_{0}} \subset \rho(B)$ and

$$
\left\|\left(B+(i s)^{\alpha} I\right)^{-1}\right\| \leq \frac{1}{\left|\operatorname{Im}\left((i s)^{\alpha}\right)\right|}=\frac{1}{\left|\sin \left(\frac{\pi \alpha}{2}\right) \| s\right|^{\alpha}} .
$$


This yields

$$
\left\|C\left(B+(i s)^{\alpha} I\right)^{-1}\right\| \leq \frac{\|C\|}{\left|\sin \left(\frac{\pi \alpha}{2}\right) \| s\right|^{\alpha}}<\frac{1}{2} .
$$

It implies that $\left(I+C\left(B+(i s)^{\alpha} I\right)^{-1}\right)^{-1}$ exists and

$$
\left\|\left(I+C\left(B+(i s)^{\alpha} I\right)^{-1}\right)^{-1}\right\| \leq \frac{1}{1-\left\|C\left(B+(i s)^{\alpha} I\right)^{-1}\right\|} \leq \frac{1}{1-\frac{\|C\|}{\left|\sin \left(\frac{\pi \alpha}{2}\right) \| s\right|^{\alpha}}} \leq 2 .
$$

Since $B$ commutes with $C$, we have the identity $A+(i s)^{\alpha} I=\left(B+(i s)^{\alpha} I\right)\left(C\left(B+(i s)^{\alpha} I\right)^{-1}+I\right)$. It shows that $\left\{(i s)^{\alpha}\right\}_{|s|>s_{0}} \subset \rho(A)$ and

$$
\left(B+(i s)^{\alpha} I\right)^{-1}\left(C\left(B+(i s)^{\alpha} I\right)^{-1}+I\right)^{-1}=\left(C+\left(B+(i s)^{\alpha} I\right)\right)^{-1}=\left(A+(i s)^{\alpha} I\right)^{-1},
$$

holds. Therefore

$$
\left\|\left(A+(i s)^{\alpha} I\right)^{-1}\right\| \leq\left\|\left(B+(i s)^{\alpha} I\right)^{-1}\right\|\left\|\left(C\left(B+(i s)^{\alpha} I\right)^{-1}+I\right)^{-1}\right\| \leq \frac{2}{\left|\sin \left(\frac{\pi \alpha}{2}\right) \| s\right|^{\alpha}}
$$

for each $|s|>s_{0}$.

In case $C \equiv 0$ we obtain the following result that has own interest.

Corollary 3.5 Let $A$ be a selfadjoint operator with domain $D(A)$ defined on a Hilbert space $H$. Let $1<p \leq 2$. If $\in L_{2 \pi}^{p}(\mathbb{R}, H)$ satisfies that $\int_{0}^{2 \pi} f(t) d t \in \operatorname{Ran}(A)$, then the conclusions of Theorem 3.2 hold for all $\frac{1}{p}<\alpha \leq \frac{2}{p}$.

The case of Banach spaces is considered in the next result.

Theorem 3.6 Let $A=B+C$, where $B$ is sectorial with spectral angle $\phi_{B} \in(0, \alpha \pi / 2)$, where $\frac{1}{p}<\alpha \leq \frac{2}{p}, 1<p \leq 2$, and let $C \in \mathcal{B}(X)$ be such that $C$ commutes with $B$. Iff $\in L_{2 \pi}^{p}(\mathbb{R}, X)$ satisfies that $\widehat{f}_{m} \in \operatorname{Ran}\left(A+(i m \omega)^{\alpha} I\right)$ for $|m| \leq 2^{1 / \alpha}\|C\|^{1 / \alpha}, m \in \mathbb{Z}$, then the conclusions of Theorem 3.2 hold.

Proof Let $s \in \mathbb{R}$ be such that $|s|>s_{0}:=2^{1 / \alpha}\|C\|^{1 / \alpha}$. Since $B$ is sectorial, $\Sigma_{\phi} \subset \rho(-B)$ for some $\phi>0$ and there exists $M>0$ such that

$$
\left\|(\lambda+B)^{-1}\right\| \leq \frac{M}{|\lambda|}, \quad \lambda \in \Sigma_{\phi}
$$

Since $\frac{1}{2}<\alpha<2$, if $\lambda=(i s)^{\alpha}:=|s|^{\alpha} e^{\frac{1}{2} \operatorname{sgn}(s) \pi i \alpha}$, then $\lambda \in \Sigma_{\phi}$ and

$$
\left\|\left((i s)^{\alpha} I+B\right)^{-1}\right\| \leq \frac{M}{|s|^{\alpha}} \quad \text { for all } 0 \neq s \in \mathbb{R} .
$$

Hence,

$$
\left\|C\left(B+(i s)^{\alpha} I\right)^{-1}\right\| \leq \frac{M\|C\|}{|s|^{\alpha}}<\frac{M}{2} .
$$


On the other hand, since $B$ and $C$ commute, we obtain that $\left\{(i s)^{\alpha}\right\}_{|s|>s_{0}} \subset \rho(A)$ and identity (9) as in the proof of Theorem 3.4. Therefore

$$
\begin{aligned}
\left\|\left(A+(i s)^{\alpha} I\right)^{-1}\right\| & \leq\left\|\left(B+(i s)^{\alpha} I\right)^{-1}\right\|\left\|\left(C\left(B+(i s)^{\alpha} I\right)^{-1}+I\right)^{-1}\right\| \\
& \leq \frac{M}{|s|^{\alpha}}\left\|\left(C\left(B+(i s)^{\alpha} I\right)^{-1}+I\right)^{-1}\right\|
\end{aligned}
$$

for each $|s|>s_{0}$.

We consider the following two cases:

(i) If $0<M \leq 1$, then $\left\|C\left(B+(i s)^{\alpha} I\right)^{-1}\right\|<\frac{1}{2}$, $|s|>s_{0}$.

(ii) If $M>1$, then, dividing by $M$ in (10), we obtain

$$
(1 / M)\left\|C\left(B+(i s)^{\alpha} I\right)^{-1}\right\|<\frac{1}{2}, \quad|s|>s_{0} .
$$

If we define for $\delta \in\{1, M\}$ the norm $\|\cdot\|_{\delta}:=(1 / \delta)\|\cdot\|$, we have

$$
\left\|C\left(B+(i s)^{\alpha} I\right)^{-1}\right\|_{\delta}<\frac{1}{2}, \quad|s|>s_{0} .
$$

Therefore, for all $0 \neq s \in \mathbb{R},\|C\|<|s|$, there exists $\left(I+C\left(B+(i s)^{\alpha} I\right)^{-1}\right)^{-1}$ and

$$
\left\|\left(I+C\left(B+(i s)^{\alpha} I\right)^{-1}\right)^{-1}\right\|_{\delta} \leq \frac{1}{1-\left\|C\left(B+(i s)^{\alpha} I\right)^{-1}\right\|_{\delta}} \leq 2 .
$$

Hence

$$
\left\|\left(I+C\left(B+(i s)^{\alpha} I\right)^{-1}\right)^{-1}\right\| \leq 2 M .
$$

Inserting the above inequality in (11), we obtain

$$
\left\|\left(A+(i s)^{\alpha} I\right)^{-1}\right\| \leq \frac{2 M^{2}}{|s|^{\alpha}}, \quad|s|>s_{0} .
$$

Again, the special case $C \equiv 0$ gives the next corollary.

Corollary 3.7 Let A be a sectorial operator with spectral angle $\phi_{A} \in(0, \alpha \pi / 2)$ where $\frac{1}{p}<$ $\alpha \leq \frac{2}{p}, 1<p \leq 2$. Assume that $f \in L_{2 \pi}^{p}(\mathbb{R}, X)$ satisfies that $\int_{0}^{2 \pi} f(t) d t \in \operatorname{Ran}(A)$, then the conclusions of Theorem 3.2 hold.

\section{Acknowledgements}

C. Lizama thanks DICYT (USACH) for financial support.

Funding

J. Bravo is partially supported by ANID-PFCHA/Doctorado Nacional/2019-21190764. C. Lizama is partially supported by ANID-FONDECYT/1180041.

Availability of data and materials

Not applicable 
Authors' contributions

The authors declare that they have equally contributed to the preparation of the paper. All authors read and approved the final manuscript.

\section{Publisher's Note}

Springer Nature remains neutral with regard to jurisdictional claims in published maps and institutional affiliations.

Received: 6 December 2020 Accepted: 14 May 2021 Published online: 22 May 2021

\section{References}

1. Atanackovic, T.M., Stankovic, S.: Dynamics of a viscoelastic rod of fractional derivative type. Z. Angew. Math. Mech. 82(6), 377-386 (2002)

2. Belmekki, M., Nieto, J.J., Rodriguez-López, R.: Existence of periodic solution for a nonlinear fractional differential equation. Boundary Value Problems 2009, 324561 (2009)

3. Bu, S.: Well-posedness of equations with fractional derivative. Acta Math. Sin. Engl. Ser. 26(7), 1223-1232 (2010)

4. Bu, S.: Well-posedness of equations with fractional derivative via the method of sum. Acta Math. Sin. Engl. Ser. 28(1), 37-44 (2012)

5. Bu, S., Cai, G.: Well-posedness of degenerate differential equations with fractional derivative in vector-valued functional spaces. Math. Nachr. 290(5-6), 726-737 (2017)

6. Bu, S., Cai, G.: Well-posedness of fractional degenerate differential equations in Banach spaces. Fract. Calc. Appl. Anal. 22(2), 379-395 (2019)

7. Bu, S., Cai, G.: Periodic solutions of fractional degenerate differential equations with delay in Banach spaces. Isr. J. Math. 232, 695-717 (2019)

8. Da Prato, G., Grisvard, P.: Sommes d'opérateurs linéaires et équations différentielles opérationnelles. J. Math. Pures Appl. 54, 305-387 (1975)

9. Denk, R., Hieber, M., Pruss, J.: R-boundedness, Fourier multipliers and problems of elliptic and parabolic type. Mem. Am. Math. Soc. 166, Article ID 788 (2003)

10. Dixmier, J.: General Topology. Undergraduate Texts in Mathematics. Springer, New York (1984)

11. Dyachenko, M., Nursultanov, E., Kankenova, A.: On summability of Fourier coefficients of functions from Lebesgue space. J. Math. Anal. Appl. 419(2), 959-971 (2014)

12. Garrappa, R., Kaslik, E., Popolizio, M.: Evaluation of fractional integrals and derivatives of elementary functions: overview and tutorial. Mathematics 7, 407 (2019)

13. Goodrich, C., Lizama, C.: A transference principle for nonlocal operators using a convolutional approach: fractional monotonicity and convexity. Isr. J. Math. 236, 533-589 (2020)

14. Gourdon, X.: Les maths en tete: Analyse, 2nd edn. Ellipses (2008)

15. Haase, M.: The functional calculus for sectorial operators. In: The Functional Calculus for Sectorial Operators. Operator Theory: Advances and Applications, vol. 169. Birkhäuser, Basel (2006)

16. Haraux, A.: Nonlinear Evolution Equations - Global Behavior of Solutions. Lecture Notes in Mathematics, vol. 841. Springer, Heidelberg (1981)

17. Hayat, T., Nadeem, S., Asghar, S.: Periodic unidirectional flows of a viscoelastic fluid with the fractional Maxwell model. Appl. Math. Comput. 151(1), 153-161 (2004)

18. Keyantuo, V., Lizama, C.: A characterization of periodic solutions for time-fractional differential equations in UMD spaces and applications. Math. Nachr. 284(4), 494-506 (2011)

19. Khan, K.N., Lamb, W., McBride, A.C.: Fractional calculus of periodic distributions. Fract. Calc. Appl. Anal. 14(2), 260-283 (2011)

20. Kilbas, A., Samko, S., Marichev, O.: Fractional Integrals and Derivatives: Theory and Applications. Gordon and Breach, New York (1993)

21. Kilbas, A., Srivastava, H., Trujillo, J.: Theory and Applications of Fractional Differential Equations. North-Holland Mathematics Studies, vol. 204. Elsevier, Amsterdam (2006)

22. Lizama, C., Poblete, V.: Periodic solutions of fractional differential equations with delay. J. Evol. Equ. 11, 57-70 (2011)

23. Nadeem, S.: General periodic flows of fractional Oldroyd-B fluid for an edge. Physics Letters, Section A: General, Atomic and Solid State Physics 368(3-4), 181-187 (2007)

24. Ortigueira, M.D., Machado, J.T., Trujillo, J.J.: Fractional derivatives and periodic functions. Int. J. Dyn. Control 5(1), 72-78 (2017)

25. Rogosin, S., Dubatovskaya, M.: Letnikov vs. Marchaud: a survey on two prominent constructions of fractional derivatives. Mathematics 6 (2018)

26. Tavazoei, M.S.: A note on fractional-order derivatives of periodic functions. Automatica 46(5), 945-948 (2010)

27. Tavazoei, M.S., Haeri, M.: A proof for nonexistence of periodic solutions in time invariant fractional order systems. Automatica 45(8), 1886-1890 (2009)

28. Winfree, A.T.: The Geometry of Biological Time. Springer, Berlin (1980) 